\title{
Endoscopic vein harvesting: the growing role in cardiac surgery
}

Pouya Nezafati ${ }^{1}$, Mahdi Kahrom², Mohammad Hassan Nezafati ${ }^{2}$

\author{
${ }^{1}$ Cardiac Rehabilitation Research Center, Isfahan Cardiovascular Research Institute, \\ Isfahan University of Medical Sciences, Isfahan, Iran \\ ${ }^{2}$ Department of Cardiac Surgery, Ghaem Hospital, Mashhad University of Medical \\ Sciences, Mashhad, Iran
}

Submitted: 26 June 2017

Accepted: 21 July 2017

Arch Med Sci Atheroscler Dis 2017; 2: e34-e36

DOI: https://doi.org/10.5114/amsad.2017.70500

Copyright (c) 2017 Termedia \& Banach

More than 300,000 coronary artery bypass grafting (CABG) operations are performed in North America annually, and it is one of the most discussed and best-evaluated surgical procedures worldwide.

The great saphenous vein (GSV) remains the most commonly used conduit for CABG due to its ease of harvesting, ready availability, versatility, resistance to spasm, and thoroughly studied long-term results.

In traditional methods of GSV harvesting, large incisions must be made; however, painful incision, dysmobility, wound complications, prolonged hospital stay, and cosmetically unappealing results may occur. Wound complications are observed in $2-24 \%$ of cases. They are associated with increased morbidity and mortality and impose a great economic burden on the health-care system [1].

In the mid 1990s, endoscopic vein harvesting (EVH) techniques were introduced as an alternative to large, incision-based open vein harvesting $(\mathrm{OVH})$ to reduce postoperative discomfort and wound complications associated with the procedure [2] (Figure 1).

Currently, EVH is growing in popularity and is the method of choice in many centers worldwide as it is associated with lower post-surgical complication rates compared to the $\mathrm{OVH}$ method [1]. The main advantage of the EVH technique is reducing the wound area, leading to improved cosmetic results and decreased incidence of lower limb morbidity, related to cellulitis, wound infection, hematoma, seroma, lymphedema, lymphorrhea, saphenous neuropathy and neuralgia, which also maintains the cost-effectiveness of EVH [3].

Although long-term graft patency following EVH has been questioned in a few reports, more recent cohort studies have reported that this technique is equally safe and effective [1, 4-6].

After proper positioning of the patient and identifying the location of the vein, a small incision is made $1.5-2.5 \mathrm{~cm}$ below/above the knee to build the entrance of the probe, which then continues its path toward the groin region. In the EVH technique it is remarkably easier to harvest the vein from the thigh due to the size and positioning of the endoscopic equipment and hence the ease of access to the GSV. Through this minimal incision, $\mathrm{CO}_{2}$ insufflation is then commenced at $12 \mathrm{~mm} \mathrm{Hg}$ of pressure with a $3 \mathrm{l} / \mathrm{min}$ flow rate to create a tunnel inside the leg $[1,6]$.

After introducing the dissection tip cannula inside the tunnel, circumferential blunt dissection of the GSV is performed along the posterior

\author{
Corresponding author: \\ Mohammad Hassan Nezafati \\ Department of Cardiac Surgery \\ Ghaem Hospital \\ Mashhad University \\ of Medical Sciences \\ Mashhad, Iran \\ PO Box: 91878544 \\ Phone: +989151578421 \\ Fax: +985136078099 \\ E-mail: mhnezafati@yahoo. \\ com
}




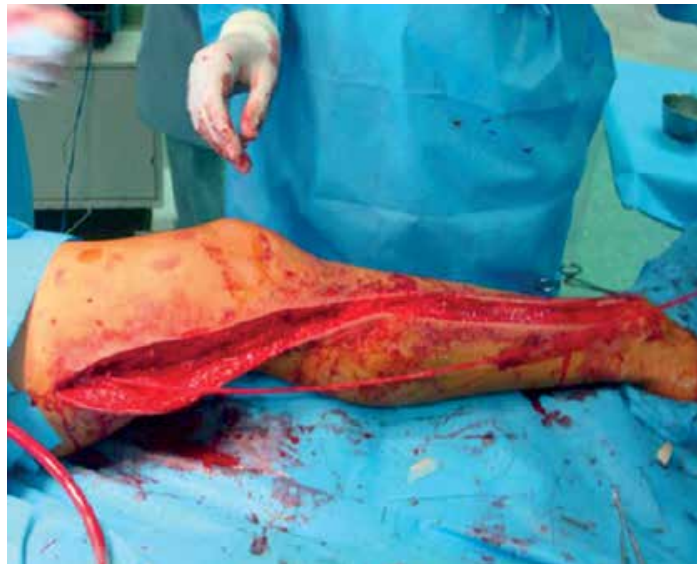

Figure 1. Comparison of $\mathrm{OVH}$ and $\mathrm{EVH}$ techniques

and lateral aspects throughout its length, after which the tributary branches are isolated and divided with bipolar electrocautery and scissors.

A tiny puncture incision is then made near the groin to clamp, ligate and divide the distal end of the GSV and remove the vein, which is checked for leakage. The branches are doubly clipped with titanium clips and avulsions are repaired with fine monofilament suture material (Figure 1). Immediately after the EVH procedure, the lower limb is tightly bandaged, and elastic stockings are used in all patients after the operation.

A considerable personal and institutional learning curve is needed upon adoption of the EVH technique as the standard of care. Macroscopic conduit lesions, such as torn tributary branches or holes requiring suture repairs, occur 3-5 times more frequently in the EVH than the $\mathrm{OVH}$ technique [7].

Some surgeons believe that the EVH technique may destroy the greater saphenous vein endothelium and it could be associated with veingraft failure, so still they have some doubts about adopting EVH.

In recent years a large quantity of evidence has emerged in the form of observational studies and randomized controlled trials, as well as meta-analyses and systematic reviews, revealing that EVH can be safely and reliably used as an alternative to OVH techniques in CABG $[4,5,7]$.

The authors have experience with more than two thousand EVH procedures in CABG patients. This research team in their previous study found that EVH has fewer postoperative wound complications and less postoperative pain in comparison with $\mathrm{OVH}$ [8]. Moreover, in our recent study we compared the immunohistochemistry (IHC) of von Willebrand factor (VWF), endothelial cadherin, caveolin and endothelial nitric oxide synthase (eNOS) between two groups of EVH and OVH. The authors demonstrated by molecular confirmation that the EVH technique causes no endothelial damage, and no structural or cellular viabili-

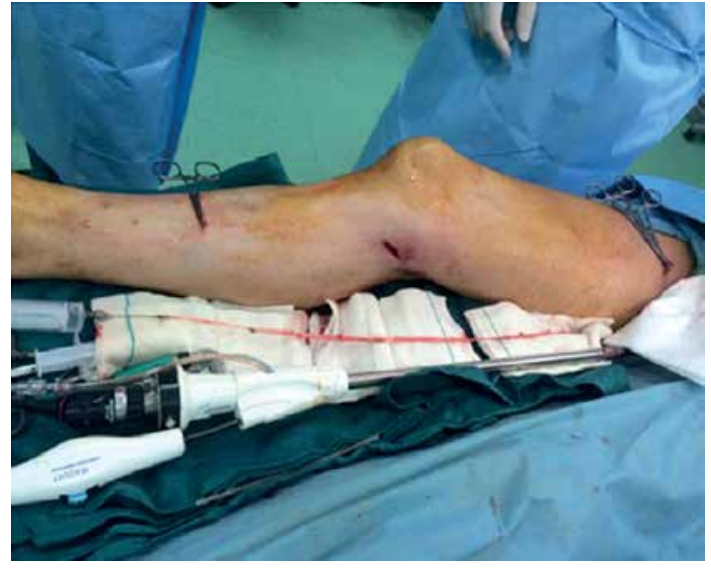

ty harm effect was found in EVH compared with $\mathrm{OVH}$. This study is in line with several trials regarding EVH [6]. These evidence-based studies confirm that vein graft quality is dependent on operator experience, and careful attention must be paid to assuring safe and reliable vein harvesting in both $\mathrm{EVH}$ and $\mathrm{OVH}$ techniques.

Furthermore, large amount of evidence has emerged in many studies confirming that the EVH technique is associated with enhanced patient satisfaction, decreased lower limb wound morbidity and improved cosmetic results.

Infective wound complications and non-infective wound healing disturbances such as wound drainage, hematoma, dehiscence, necrosis, need for surgical debridement, and seroma formation following traditional OVH occur in $2-24 \%$ of cases. The morbidity associated with these complications not only results in a significant economic and clinical burden, but also ultimately leads to re-intervention, increased local pain, impaired mobilization, and patient dissatisfaction [7].

On the other hand, surgeons' fatigue before reaching the main part of the operation is crucial; however, a great amount of time is expended on skin closure in the OVH technique, whereas EVH is performed in a satisfactorily shorter time.

In another study by Chernyavskiy et al. the authors demonstrated that the large length of incision during $\mathrm{OVH}$ can damage the lymphatic system of the lower limb, resulting in clinical manifestations of impaired lymphatic drainage in the early post-surgical period. Furthermore, the incidence of paresthesia in the lower limb increased by 4.7 times in the patients of the $\mathrm{OVH}$ group. It is reported that intensity of post-surgical pain in the leg is lower in the EVH group, with significant improvement in patients' mobilization and quality of life [1].

The reduced wound area in EVH patients leads to reduced blood loss during the procedure, which directly influences the requirement of blood trans- 
fusions and significantly reduces the number of peri-procedurally transfused red blood cell units [9].

Finally, neither the meta-analyses nor the randomized studies showed any significant difference in terms of postoperative $\mathrm{MI}$, vein graft stenosis or occlusion, angina recurrence, need for repeat revascularization or 30-day or mid-term mortality between the $\mathrm{EVH}$ and $\mathrm{OVH}$ group of patients. On the other hand, based on abundant studies, the association between EVH technique and poor vein graft patency has recently been called into question, and GSVs harvested using the endoscopic technique are of comparable quality to those obtained using the open technique $[1,7,10]$.

In conclusion, published studies comparing EVH with OVH show reduced wound-related morbidities, improved patient quality of life, shorter hospital stay, and reduced postoperative pain at the harvest site following EVH, thereby translating into increased patient satisfaction.

An observational study of patients undergoing CABG surgery at centers participating in the Society of Thoracic Surgeons (STS) national database found no evidence of an association of EVH with long-term mortality or a composite of death, $\mathrm{MI}$, or need for repeat revascularization [2].

In general, favorable results of EVH should result in more widespread use of this technology, and proper instrumentation, procedural training and technical expertise of the personnel involved in the EVH process are of vital importance to preserve the GSV as a truly viable and durable bypass conduit.

\section{Conflict of interest}

The authors declare no conflict of interest.

\section{References}

1. Chernyavskiy A, Volkov A, Lavrenyuk O, Terekhov I, Kareva Y. Comparative results of endoscopic and open methods of vein harvesting for coronary artery bypass grafting: a prospective randomized parallel-group trial. J Cardiothorac Surg 2015; 10: 163.

2. Williams JB, Peterson ED, Brennan JM, et al. Association between endoscopic vs open vein-graft harvesting and mortality, wound complications, and cardiovascular events in patients undergoing CABG surgery. JAMA 2012; 308: 475-84.

3. Hussaini BE, Lu XG, Wolfe JA, Thatte HS. Evaluation of endoscopic vein extraction on structural and functional viability of saphenous vein endothelium. J Cardiothorac Surg 2011; 6: 82.

4. Lopes RD, Hafley GE, Allen KB, et al. Endoscopic versus open vein-graft harvesting in coronary-artery bypass surgery. N Engl J Med 2009; 361: 235-44.

5. Zenati MA, Shroyer AL, Collins JF, et al. Impact of endoscopic versus open saphenous graft harvest technique upon CABG patient outcomes in the randomized ROOBY trial. J Thorac Cardiovasc Surg 2011; 141: 338-44.

6. Nezafati MH, Nezafati P, Amoueian S, Attaranzadeh A, Rahimi HR. Immunohistochemistry comparing endo- scopic vein harvesting vs. open vein harvesting on saphenous vein endothelium. J Cardiothorac Surg 2014; 9: 101.

7. Raja SG, Sarang Z. Endoscopic vein harvesting: technique, outcomes, concerns and controversies. J Thorac Dis 2013; 5: S630-7.

8. Nezafati MH, Nezafati P. Descriptive analysis of endoscopic versus traditional open vein harvest technique for coronary artery bypass graft surgery: report of 1974 casess. Iran Heart J 2014; 14: 17-22.

9. Andreas M, Wiedemann D, Stasek S, et al. Endoscopic vein harvesting is influenced by patient-related risk factors and may be of specific benefit in female patients. Interact Cardiovasc Thorac Surg 2013; 17: 603-7.

10. Sastry P, Rivinius R, Harvey R, et al. The influence of endoscopic vein harvesting on outcomes after coronary bypass grafting: a meta-analysis of 267,525 patients. Eur J Cardiothorac Surg 2013; 44: 980-9. 